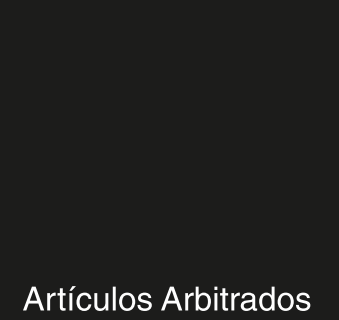

\title{
La disputa por la ciudad: el rol de los actores sociales en la gobernanza del suelo urbano en la ciudad de Córdoba (Argentina)
}

\author{
Daniela Mariana Gargantini
}

Doctora en Arquitectura por la Universidad Nacional de Rosario (Argentina) y magíster en Hábitat y Vivienda (Mar del Plata, Argentina). Investigadora adjunta del CONICET y miembro del equipo técnico del Centro Experimental de la Vivienda Económica (Córdoba). Desde 2004 se desempeña como profesora titular de la cátedra Problemática socio-habitacional y encargada del Servicio socio-habitacional de la Facultad de Arquitectura de la Universidad Católica de Córdoba, Argentina. Directora de la Especialización en Gestión Integral del Hábitat de la Facultad de Arquitectura de la Universidad Católica de Santa Fe, Argentina (UCSF). Docente titular en asignaturas de grado y posgrado en Córdoba, Santa Fe, Chaco y Deusto (Bilbao, España). Desde 2007 coordina la Red de Responsabilidad Social Universitaria de la Asociación de Universidades Jesuitas de América Latina. dmgargantini@gmail.com.

\section{Natalí Peresini}

Es arquitecta por la Universidad Nacional de Córdoba y becaria doctoral por el CONICET integrando el equipo técnico del Centro Experimental de la Vivienda Económica (Córdoba). Miembro del grupo de investigación "Estrategias de superación de conflictos urbanos y violaciones al derecho a la ciudad" de la Facultad de Arquitectura de la UCC y de la investigación SECyT 2016-2017 FAUD-UNC "La escala territorial en la configuración de las periferias urbanas. Lógicas espaciales y ordenamiento específico en el sector este de la ciudad de Córdoba”. Miembro de la Red Ciudadana Nuestra Córdoba, con diferentes colaboraciones en el grupo de trabajo sobre desarrollo urbano. nataliperesini@ceve.org.ar. 


\begin{abstract}
Resumen
La ciudad se instituye como locus de los conflictos urbanos evidenciando las disputas de poder sobre su territorio. El acceso al suelo constituye uno de los procesos de mayor conflictividad en la consecución de un hábitat digno y de inclusión en la ciudad, por lo que considerar las representaciones y redes de interacción de los agentes involucrados favorece y potencia la comprensión de la producción física y simbólica de la ciudad. En el presente artículo se exponen, desde un enfoque cualitativo, los avances de la investigación en curso, referida al estudio del gobierno del suelo urbano en la ciudad de Córdoba (Argentina), a partir de la indagación y confrontación de las representaciones de actores sociales comunitarios influyentes, sus lógicas de comprensión y acción, así como las relaciones de articulación en torno a este conflicto urbano.
\end{abstract}

\title{
Palabras clave
}

Gobernanza de suelo urbano; actores comunitarios; representaciones; conflictos urbanos.

\section{Abstract}

The conflict for the city: the role of social agents in urban land governance in the city of Córdoba (Argentina)

The city is instituted as the locus of the urban conflicts, exposing the power disputes over its territory. Access to the urban land is one of the processes of greater conflict in the achievement of a decent habitat and inclusion in the city, in which case the consideration of the networks of interaction of the agents involved, facilitates and enhances the understanding of the physical and symbolic production of the city.

This paper presents, from a qualitative perspective, the advances of research related to the study of urban land governance in the city of Córdoba (Argentina), based on understanding and confronting the representations of different social agents as they influence their logics of understanding, as well as the relations of articulation around this urban conflict.

\section{Keywords}

Governance of urban land; agents; representations; urban conflicts. 


\section{La disputa por la ciudad:}

el rol de los actores sociales en la gobernanza del suelo urbano en la ciudad de Córdoba (Argentina)

\section{Introducción}

Desde el marco conceptual provisto por LefeVBre (1978), la producción del espacio urbano es entendida a partir de la construcción socio-política del territorio, teniendo en cuenta las prácticas espaciales - la producción material y las formas en que se genera, utiliza y percibe el espacio-, las representaciones del espacio —el espacio concebido desde los saberes técnicos y racionales hegemónicos-y, por último, los espacios de representación, es decir, los espacios vividos que se construyen entre las dinámicas simbólicas y las experiencias espaciales. Los actores que participan de diferentes maneras en esta producción poseen distintas representaciones de lo vivido y de los modos de producción del espacio. Estas pueden comprenderse dentro del concepto de representaciones sociales elaborado por MoscovicI (1979), entendidas como un corpus organizado de conocimientos gracias al cual los hombres hacen inteligible la realidad física y social, refiriéndose a una forma de pensamiento que sintetiza explicaciones acerca de cómo cada grupo social piensa y organiza su vida, constituyendo modalidades de pensamiento práctico orientadas a la comunicación, la comprensión y el dominio del entorno social, material e ideal (JoDELET, 1986).

En el proceso histórico de producción de la ciudad, las maneras de pensarla fueron variando. Nos interesa destacar aquí el renovado debate que supuso la adopción por distintas organizaciones (principalmente sociales) de la CarTa Mundial por el Derecho a la CiUdad en el año 2004, la cual revitalizó el planteo sobre el Derecho a la Ciudad esbozado por LEFEBVRE (1969) en décadas anteriores. En este sentido, se reconoció la urgencia de la construcción de ciudades más justas, equitativas y sustentables en un sentido integral que contemple múltiples dimensiones, entre ellas el derecho al suelo y la vivienda, al transporte público, a un medioambiente sano, así como el goce de otros derechos sociales, económicos, culturales y ambientales previstos en las distintas convenciones relacionadas con los derechos humanos.

Desde este marco, la ciudad se interpreta como locus de los conflictos urbanos, y para analizarla se precisa identificar y profundizar en el conocimiento de los actores que intervienen en ella, a partir de las estrategias y relaciones que despliegan en la disputa del poder sobre el territorio que habitan. Así, en toda ciudad se distinguen conflictos, poderes y resistencias que necesitan ser identificados y reconocidos desde su visibilización y problematización, como primer paso para su resolución. Este es el objetivo y la clave de la investigación que da origen al presente artículo. 
1. Para acceder a los conceptos trasladados se puede consultar en la página Web de ONU-HÁBITAT: http:// es.unhabitat.org/ gobernanza/ (consulta: 16/06/2017).

2. Chantal Mouffe, en su libro En torno a lo político (2009), expresa que "lo político" involucra la dimensión de antagonismo que considera constitutiva de las sociedades humanas, diferenciándolo de "la política" (entendiendo esta última como el conjunto de prácticas e instituciones a través de las cuales se crea un determinado orden, organizando la coexistencia humana en el contexto de la conflictividad derivada de lo político).
El Estado y el rol de los actores sociales en relación con la gobernanza del suelo urbano

La gobernanza urbana, según ONU-HÁBITAT ${ }^{1}$, puede definirse como las diversas formas en las que las instituciones y los individuos se organizan en la gestión cotidiana de una ciudad tomando en cuenta los procesos utilizados para llevar adelante la agenda urbana, a corto, mediano y largo plazo. Ahora bien, hablar de gobierno del suelo urbano supone avanzar hacia una definición propia del Estado y su relación con el mercado. Sin olvidar la importancia de reconocer la necesidad de mecanismos de participación ciudadana y de articulación público-privada en el diseño como en la implementación de políticas públicas, y lejos de la adhesión a referencias al viejo poder jerárquico y a las concepciones centralistas, hablar de gobierno del suelo urbano supone otorgarle al Estado y al sector gubernamental un rol esencial en la planificación, regulación y gestión del uso del suelo. En este rol es central su liderazgo en la dinámica política de la ciudad (SuBIRATS y BuRGUÉ, 2005), así como la capacidad técnica y de gestión del municipio en el manejo de lo urbano, a fin de evitar lo que menciona Dufour (2009) como la "privatización de la decisión pública”.

Así como el Estado, quienes ostentan el poder sobre el territorio intentan organizar e institucionalizar prácticas sociales, empresarias e incluso políticas bajo su influencia en favor de los intereses particulares que los movilizan. Al mismo tiempo, se produce la organización del contrapoder (CASTELLS, 2012), a través de movimientos sociales capaces de instituirse como agentes contrahegemónicos. En este aspecto, cabe diferenciar dos tipos de movimientos sociales: los que aspiran a tomar el poder y los que niegan esta capacidad. Los primeros se convierten en movimientos políticos, mientras que los segundos buscan impactar en las personas y en las categorías culturales en las que se piensa la sociedad. Estos últimos son los que reivindican la idea de intervención ciudadana a través de la participación política, entendida no como movimiento o partido, sino como "lo político" ${ }^{2}$ inherente al ser ciudadano.

Aunque es difícil delimitar la multiplicidad de actores que se disputan la ciudad, el criterio de corte compartido en este trabajo adhiere al planteo de BoRJA (1975), al reconocer a los actores que disputan cuestiones que tienen que ver específicamente con la organización de la producción y consumo del territorio, así como a las reglas e instituciones que regulan dichas cuestiones. En esta concepción, un aspecto importante resulta el conocimiento de los actores involucrados en estas situaciones de conflicto y cuáles son sus representaciones; de 


\section{La disputa por la ciudad:}

el rol de los actores sociales en la gobernanza del suelo urbano en la ciudad de Córdoba (Argentina)

allí la importancia de tener en cuenta las diferentes formas en que estos actores representan o construyen los conflictos. Para ello, desde la investigación que sustenta este trabajo, se intentó contribuir en el reconocimiento de los actores sociales más influyentes en la gobernanza del suelo de la ciudad de Córdoba, y junto con ello, en la identificación y reconocimiento de las distintas representaciones que estos agentes tienen de los conflictos urbanos, su priorización, capacidad de negociación y articulación con otros agentes estratégicos para ellos y las posibles soluciones o intervenciones sugeridas ante los mencionados conflictos.

Desde este marco conceptual, se entiende que existen situaciones de conflicto y violaciones al Derecho a la Ciudad cuando se generan situaciones “objetivas” pero también "subjetivas" de vulneración de estos derechos. Es decir, tal como lo expresa Azuela (2009), las situaciones de conflictividad pueden producirse también por las representaciones sociales que tienen los diferentes actores urbanos respecto de la insatisfacción, incumplimiento o falta de atención de cuestiones contempladas o no dentro de los marcos normativos y operativos formales. Para el estudio realizado, los conflictos urbanos se conciben como "tensiones, en estado latente o manifiesto, no esporádicas, existentes entre dos o más agentes individuales $u$ organizados (de carácter público, privado y/o de la sociedad civil), vinculadas al soporte físico de la ciudad, producto de la violación de derechos colectivos explicitados en la Carta Mundial por el Derecho a la Ciudad" (Gargantini y Martiarena, 2016 ).

Si bien la investigación abarcó la consideración de los diferentes actores que intervienen en la producción de la ciudad (actores estatales - políticos y burocracia-, de municipio y provincia, actores económicos o empresariales -individuales y organizaciones sectoriales- y actores comunitarios — representantes de organizaciones sociales - [HERzER y Pírez, 1993]), la publicación se centrará en identificar y analizar las representaciones de actores comunitarios organizados representativos en la lucha por el acceso al suelo de la ciudad de Córdoba, así como en la heterogeneidad de relaciones, intereses y capitales puestos en juego que afectan y definen los sentidos predominantes de su acción sobre el espacio urbano.

\section{Marco metodológico de abordaje}

Frente al marco conceptual y contextual expuesto, se presentará el estudio sobre el gobierno del suelo urbano en Córdoba capital (Argentina), que forma parte de proyectos de 
3. "Conflictos urbanos y violaciones al derecho a la ciudad en Córdoba, Argentina" (2012-2014)

$y$ "Detección priorizaday proposición de estrategias de superación de conflictos urbanos y violaciones al derecho a la ciudad en Córdoba capital" (20142016) Financiación: SIV-

Universidad Católica de Córdoba. Dirección: Dra. Arq. Daniela Gargantini y Mgtr. Arq. Miguel Martiarena. Miembros: Dra. Desirée D'Amico, Mgtr. Joaquín Peralta, Arq. Verónica Greppi, Mgtr. Pamela Cáceres, Lic. Hugo Romero, Lic. Paula Reynoso, Lic. Evelyn Cels. Carolina Nievas, Mgtr. Gustavo Re, Arq. Natali

Peresini, Arq. Lucía Fank, Arq. Mónica Alvarado, Arq. Paola Sánchez y Mgtr. María Cerrezuela. investigación que buscaron localizar, caracterizar y comprender los conflictos urbanos entendidos como violaciones al Derecho a la Ciudad, a fin de contribuir desde su visibilización y comprensión a la proposición de estrategias de superación de dichos conflictos ${ }^{3}$. La pesquisa parte de la indagación de las representaciones de agentes sociales involucrados en dicho gobierno, sus lógicas de comprensión y acción, así como las relaciones de articulación en torno a los conflictos relacionados con su acceso y uso.

Desde el presente artículo se intentará contribuir a la identificación —desde un abordaje cualitativo- de las representaciones de los actores comunitarios organizados más influyentes en la gobernanza del suelo de la ciudad de Córdoba sobre los conflictos urbanos existentes, su priorización, capacidad de negociación-vinculación con otros agentes estratégicos y las posibles soluciones o intervenciones que podrían tomarse ante los mencionados conflictos. Se busca hacerlo en relación con el acceso, uso y control del suelo, dado que constituye un recurso estratégico en el derecho a un hábitat digno.

Para dar respuesta al objetivo de indagar y analizar críticamente la manera en que se gobierna el suelo urbano en la ciudad, se definieron dimensiones y categorías analíticas teóricas y emergentes, sobre las cuales se estructuró el estudio. A saber:

- Principales conflictos existentes: listado priorizado de situaciones de tensión identificadas en la ciudad, tanto en estado latente como manifiesto.

- Principales sectores afectados: diferentes grupos poblacionales, zonas o barrios de la ciudad que se encuentran directa o indirectamente influidos por los resultados o impactos producidos por los conflictos mencionados.

- Causas de los conflictos priorizados: fundamento, origen, motivo o razón de la existencia y manifestación de los conflictos.

- Valoración de la intervención gubernamental (en sus tres niveles: nacional, provincial y municipal): apreciación positiva o negativa de la orientación del actuar gubernamental, como de las diferentes materializaciones de dicho accionar (políticas, modos de gestión, instrumentos y normativas empleados por los diferentes niveles del gobierno en torno a las problemáticas).

- Prácticas de resolución/superación de los conflictos: representaciones sobre el modo y tipo de intervenciones sugeridas para solucionar las problemáticas.

- Tipos de vinculaciones o redes existentes entre distintos actores: conjunto de actores vinculados a través de una relación o un conjunto de relaciones sociales; indagación de quién/es 


\section{La disputa por la ciudad:}

el rol de los actores sociales en la gobernanza del suelo urbano en la ciudad de Córdoba (Argentina)

y cómo se gobierna el suelo en la ciudad, a partir de las representaciones sobre quiénes son los agentes más influyentes, las razones por las que lo son y el grado y tipo de relaciones interactorales existentes (contacto frecuente, mayor confianza, intercambio de información y relaciones conflictivas).

- Valoración de las instituciones especializadas: distintos sujetos individuales o colectivos referentes en la temática de estudio. Conocimiento de los entrevistados, contactos o vinculaciones virtuosas con dichos referentes en busca de estrategias de superación de los conflictos.

A partir de estas dimensiones y categorías, se diseñó el instrumento de relevamiento base (entrevista semiestructurada), para indagar las representaciones de los agentes involucrados en la gestión urbana. Para identificar los agentes que entrevistar vinculados con el acceso, control y gestión del suelo urbano, se elaboró una muestra cualitativa de tipo intencional (GUBER, 1991). La selección de los entrevistados —más allá de la accesibilidad para la realización de las entrevistas - se dio sobre la base del reconocimiento previo de actores significativos en la gobernanza del suelo urbano, según información proporcionada por informantes clave (método Delphi). Estos últimos fueron en su mayoría especialistas que regularmente trabajan temáticas urbanas y relativas al acceso al suelo, así como referentes de organizaciones sociales que interactúan con frecuencia con otros actores vinculados con el acceso al suelo urbano.

Junto a los actores estatales y económicos, los actores comunitarios entrevistados ${ }^{4}$ fueron los siguientes:

- Asociación Civil Amigos de la Reserva San Martín (ACASM), organización sin fines de lucro que nace y se sostiene con el objetivo de preservar uno de los mayores pulmones verdes (bosque nativo y refugio de flora y fauna nativa) de la ciudad de Córdoba: el parque municipal San Martín;

- Nuestro Hogar 3 (NH3), organización de vecinos de un asentamiento irregular de la periferia sur de la ciudad de Córdoba;

- Defendamos Alberdi (DA), multisectorial conformada por vecinos del barrio y diversas organizaciones centradas en el popular y tradicional barrio Alberdi;

- Encuentro de Organizaciones (EO), organización política no partidaria de la ciudad de Córdoba que tiene como lema "construir poder popular desde la comunidad para una vida digna”;
4. Las entrevistas se llevaron a cabo durante los últimos meses de la primera gestión del intendente Ramón Mestre (20112015). Colaboraron en la realización y transcripción de entrevistas Guadalupe Jáuregui, Martín Santos, Virginia Romanutti, Lucio Scardino, Agostina Pollano, Beatriz Valencia, Romina Raccone, Virginia Monayar, Laura Basso, Victoria Fernández, Mara Vallejos, Ignacio Pellón, Elisa Viñas y Fernanda Etchepareborda. 
- Cecopal (CEC), ONG que tiene como propósito promover y acompañar la reconstrucción o consolidación de las formas organizativas de los sectores populares;

- Foro en Defensa del Patrimonio Cultural en Córdoba (FDPC), espacio ciudadano inscripto en las orientaciones que surgen de las cartas, convenciones, acuerdos y recomendaciones vinculadas con la protección, defensa y promoción de las herencias patrimoniales que pertenecen a los ciudadanos y a la comunidad de Córdoba;

- Foro Ambiental Córdoba (FA), espacio de participación de personas e instituciones relacionadas con la temática ambiental y la participación ciudadana;

- Asentamiento Renault (AR), organización de vecinos de un asentamiento referente ubicado en la periferia sur de la ciudad de Córdoba;

- Movimiento Evita (MOV), organización política partidaria de alcance nacional;

- Foro Núñez (FN), grupo de vecinos del tradicional barrio Cerro de las Rosas, organizados para proteger el arbolado urbano de Av. Rafael Núñez;

- Red de Vecinos San Vicente (SVIC), espacio de articulación de vecinos y asociaciones del tradicional barrio San Vicente, destinado a promover y proteger el desarrollo socio-cultural de la zona;

- El Bordo (BOR), organización de vecinos de un histórico asentamiento, que se convirtieron en referentes de lucha y resistencia por haber logrado la propiedad de la tierra que ocupan; - Ceve (CEVE), centro de investigación, experimentación, desarrollo y transferencia de tecnologías de construcción y gestión integral, destinadas a la producción social del hábitat;

- Sehas (SEH) ONG destinada a servicios habitacionales y desarrollo social y

- Serviproh (SER), institución de promoción y desarrollo que colabora en la construcción colectiva de procesos sociales organizativos de sectores populares urbanos.

Para la interpretación de los datos se empleó la técnica de análisis de contenido teniendo en cuenta el criterio de saturación teórica (nivel de repitencia en el tipo de datos que ofrecían los entrevistados al indagar sobre las mismas preguntas). Esto supuso la codificación del material privilegiando la detección de categorías tanto teóricas, previamente establecidas por el grupo de trabajo, como emergentes. Se priorizó el análisis de contenidos manifiestos y latentes, poniendo atención no solo en lo explícitamente expresado, sino también en otras cuestiones subyacentes que se pudieron detectar a través de sus intervenciones. La herramienta utilizada para procesar las entrevistas fue el software Atlas Ti, que sirvió como decodificador del material producido, con la elaboración paralela de algunas relaciones entre las distintas dimensiones y categorías en estudio. 


\section{La disputa por la ciudad:}

el rol de los actores sociales en la gobernanza del suelo urbano en la ciudad de Córdoba (Argentina)

A continuación, se presenta una lectura comparada de las representaciones subyacentes en los discursos de los entrevistados. Específicamente, se presta especial atención a los elementos de sentido que los entrevistados emplearon para definir los conflictos urbanos existentes en la ciudad y el acceso al suelo como conflicto particular; al mismo tiempo se discuten los puntos de coincidencia o disidencia entre los diferentes actores contactados. Por la extensión del artículo, se transcriben solo algunas citas textuales que se consideraron representativas de las entrevistas.

Representaciones sobre el gobierno del suelo urbano en la ciudad de Córdoba

$\mathrm{Al}$ indagar sobre los tipos de conflictos priorizados, los entrevistados identificaron con mayor recurrencia la dificultad en el acceso a la tierra y a la vivienda en la ciudad desde diferentes expresiones, tales como la desigualdad en el acceso al suelo, la especulación inmobiliaria, la inseguridad en la tenencia y la preocupación por el alto grado de segregación urbana que se demuestra en las urbanizaciones aisladas - ya sean privadas o de planes de vivienda del Estado- que se han ido consolidando en los últimos años. Vinculados con ello se mencionaron procesos expulsivos de gentrificación y densificación, tomas de tierra, incremento excesivo de los costos de los terrenos, hacinamiento en los barrios populares y exclusión de los sectores más pobres.

CEVE: "La falta de respuesta del Estado a la necesidad de tierra, no solo de los asentamientos o de los sectores que toman tierras, sino de todo el sector de clase media, como se está viendo con el tema PROCREAR [...] que la gente ya directamente ya no consigue porque no hay oferta de tierra en Córdoba y acude al interior, pero es como no tener una política del Estado de provisión de tierras para un sector medio”.

FN: "La tierra siempre va a sectores ricos. Esto desplaza a los más pobres, que son a su vez los que más necesitan estar cerca. Se les encarece la vida si se los envía a la periferia, en función de la lejanía sufren más el transporte y la pérdida de tiempo”.

MOV: "Me parece que el acceso al suelo, hoy la expresión de la toma de tierra tiene que ver con una salida que no está quedando a otra, ante la falta de políticas de tierras, de acceso a 
5. Se nombró particularmente a los últimos períodos desde 1999: "los intendentes

Mestre, Giacomino, Juez y Kamerath" (ACASM). tierra desde el Estado como política pública. Los sectores trabajadores tampoco están pudiendo tener o poder acceder a la tierra, si un pedazo de tierra sale $\$ 400.000$, no está acorde a un asalariado, tampoco".

En segundo lugar, se mencionó la ausencia de planificación de la ciudad como conflicto urbano, referida a un Estado ausente, falta de regulación hacia el privado, desidia, falta de programación y organización de la ciudad en las últimas administraciones ${ }^{5}$, superposición de usos por falta de control y la planificación —u omisión de esta- para favorecer a algunos sectores empresariales que manejan la especulación como estrategia de valorización del suelo y las normativas a discreción. Extensión y segregación urbana se perciben así como consecuencias ante la desatención y abandono estatal de ciertas áreas de la ciudad donde confluyen problemáticas urbanas, habitacionales, sociales, de violencia y ambientales. Esta ausencia del Estado se identificó entonces como uno de los conflictos centrales.

ACASM: "Hay una invasión de una zona sobre otra, entonces no están bien delimitadas las áreas como para que funcione bien. Capaz que vos estás en un barrio residencial y te encajan un edificio de diez pisos en el medio, como ha pasado acá en Villa Belgrano”.

CEVE: "Es que la ciudad en definitiva la están terminando de construir las inmobiliarias [...] y se ha perdido el equilibrio que antes había, por lo menos un poco más que ahora. Y como que ahora lo único que se está modificando la estructura de la ciudad, en realidad la están modificando 'autorizadamente', entre comillas, son los emprendimientos inmobiliarios”.

DA: "Las grandes corporaciones inmobiliarias [...] han creado [...] un fondo fiduciario con el dinero que ha llegado desde el campo, o sea, la renta agraria que ha habido, que se ha volcado a la especulación inmobiliaria, no a resolver los problemas de vivienda que hay en la Argentina”.

En tercer lugar, y asociada con el conflicto anterior, surgió la falta de cobertura en los servicios básicos (agua potable, recolección de basura, cloacas-aguas servidas, tránsito) y de infraestructura mínima (educación, salud, espacios verdes).

DA: "Los que vienen a habitar los nuevos emprendimientos también sufren el tema de la infraestructura. O sea, es como que lo que dicen vender no es lo que venden, te venden otra cosa". 


\section{La disputa por la ciudad:}

el rol de los actores sociales en la gobernanza del suelo urbano en la ciudad de Córdoba (Argentina)

SEH: "Los barrios muy humildes con servicios en bastante mal estado, entonces, suponete te podés pasar, se pueden pasar meses con el tema de los desechos cloacales ahí dándole vuelta en el barrio sin atender. Entonces hay mucho de eso en los barrios precarios, ¿no? En villas y otros barrios, no solamente villas. Entonces se dilatan en el tiempo un montón de esas soluciones, no se atienden".

Como menciones particulares surgieron temáticas como la desarticulación y la escasa participación de actores sociales en la lucha por la tierra. Esta ausencia de participación se presenta también asociada o nutrida por la falta de credibilidad que estos espacios producen en la ciudadanía, lo que desalienta todo tipo de iniciativa de agrupamiento y reclamo. En este sentido, el municipio se visualiza como un gran obstaculizador en la consolidación de espacios ciudadanos.

ACASM: "Nosotros vivimos en una lucha permanente contra la municipalidad, porque la municipalidad... nosotros tenemos proyectos para proteger y mejorar la reserva y no nos dan ni cinco de bola... además son herméticos, ellos tienen la mayoría absoluta y deciden no escuchar nada y eso no puede ser".

SEH: "Otro problema gravísimo es la falta de respuesta del Estado, es decir, ante las gestiones, ordenadas, organizadas, con asesoramiento técnico, de numerosos barrios [...] ¿Qué respuesta ha tenido? ¡Nada! ¡Cero! Entonces estos dos extremos; me parece que es gravísimo en la ciudad de Córdoba".

DA: "Los canales de participación son muy leves y sobre todo poco democráticos, y terminan en connivencia con los desarrollistas. Principalmente por el Estado este, en forma piramidal que manda la línea y es muy difícil”.

En esta priorización de conflictos por parte de los actores sociales cordobeses se pudo constatar, a diferencia de los otros actores entrevistados (estatales y económicos), un reconocimiento más holístico e integral de las tensiones existentes en la ciudad. Esta mayor apertura a reconocer numerosos y complejos conflictos fuera de su ámbito de trabajo fue así distintiva. Las percepciones estuvieron marcadas por la lógica de la necesidad y por la lógica del derecho, es decir, fuertemente relacionadas con las necesidades particulares de cada una de las organizaciones y con la visión construida a partir del trabajo conjunto con 
organizaciones técnicas de apoyo a los sectores sociales (ONG) que, según las representaciones manifiestas, se constituyen en intermediarios en la construcción del hábitat, rol que en esencia debería ser asumido por el Estado.

Sobre los principales afectados, los referentes comunitarios entrevistados consideran que por tratarse de problemáticas urbanas todos los sectores de la ciudad se ven perjudicados. Sin embargo, mencionan particularmente a las clases populares con características de vulnerabilidad o con menos recursos, situándolas en barrios o zonas periféricas de la ciudad. En algunos casos se mencionó a las clases medias como afectados directos en relación con el acceso al suelo y los precios que maneja el mercado para la vivienda propia.

MOV: "El acceso al suelo está afectando a todos los sectores, de la economía formal y a sectores trabajadores también. De distintas formas, me parece que a sectores medios también. Yo creo que acá los principales afectados, me parece que está claro que los sectores de menores recursos son los que menos posibilidades tienen".

CEVE: "Afectan la estructura de la ciudad, afectan a la ciudad como tal [...], el hecho de que haya tres parcelas de 2 o $3000 \mathrm{~m}^{2}$ por familia es como un abuso del consumo de tierra, no es que perjudica a esa familia, al contrario, porque ellos viven demasiado bien, sino que perjudica la ciudad en cuanto a disponibilidades".

DA: "En realidad sufren los más débiles, o sea, es una cuestión de poder económico, sufren los más débiles".

La fragmentación urbana detectada influye también en las percepciones, preconceptos y prejuicios entre sectores sociales, y realimenta un círculo vicioso de segregación y violencia. Cabe destacar que estas expresiones de violencia no solo se perciben entre grupos de diferentes sectores sociales, sino dentro de las mismas comunidades, cuando se pierden de vista tensiones barriales preexistentes al intentar acercar soluciones desde los organismos estatales. SEH: "Porque no tenés conciencia, pero empezás a desarrollar mecanismos, pensamientos, tu concepto, tu ideología se empieza a mover en un marco... de circular. Empieza a moverse de una forma que responde a esos parámetros de división y fragmentaciones”.

En este aspecto, al igual que en la priorización de conflictos, los actores comunitarios dieron cuenta de un conocimiento minucioso de la ciudad y sus problemáticas, y de capacidad para 


\section{La disputa por la ciudad:}

el rol de los actores sociales en la gobernanza del suelo urbano en la ciudad de Córdoba (Argentina)

detallar y georreferenciar diversos conflictos con sus actores y territorios afectados. Esto no sucedió con los actores estatales y económicos entrevistados, los cuales mencionaron un "a todos" como unidad generalizadora que, al no permitir visualizar particularidades, desdibuja el foco de conflicto para convertirse en una simple expresión de situaciones urbanas naturalizadas.

Entre las intervenciones respecto de las causas que motivan los conflictos detectados, resaltó por repitencia entre los actores comunitarios la mención a la falta de planificación urbana como la principal, aun cuando también fue indicada como conflicto. Según la mayoría de los entrevistados (nueve de doce), existe una connivencia entre el Estado y el capital privado que beneficia a estos últimos sobre la población, mencionando coaliciones entre municipio y los desarrollistas o el mercado inmobiliario financiado por el capital agrario, y reforzado la nula planificación o la falta de respeto a los planes de ordenamiento existentes.

MOV: “[...] me parece que hay una falta, en la última década, de una política pública que regule el acceso a la tierra. [...] Que los precios se disparen y que el Estado no pueda regular los precios de la tierra tampoco. Y que las últimas gestiones, tanto provinciales como municipales, han tenido entre sus representantes a los intereses de los grupos desarrollistas”.

NH3: "Las causas, la desidia de todos los que están como autoridades".

EO: "Hay una coalición de la provincia con el capital agrario de los pooles de siembra, que son los que movilizan el mercado inmobiliario. El mercado está regulado a favor del privado, dejando a la clase media más consolidada el acceso a la tierra solo a través de políticas habitacionales crediticias".

En segundo orden, pero continuando la misma línea de apreciación, se expresó como causa un gobierno local débil, con ineficiencia técnica e incapacidad para el mantenimiento de servicios básicos e infraestructura, planificación y manejo de las relaciones entre los actores. Esta causa priorizada hace foco en el Estado como principal responsable por la desidia, por el desentendimiento de la regulación del suelo, la falta de políticas públicas respecto del acceso a la tierra y a la vivienda y por la falta de cumplimiento legislativo. 
Como emergente surgió la apreciación de algunos actores sociales, de entender como fundamental causante de los conflictos la falta de interés en dar lugar a una participación ciudadana real por parte de la actual administración. La estructura piramidal del Estado, que dificulta acceder con transparencia a la información a los sectores de la sociedad civil, se percibe simultáneamente como facilitadora de la asociación Estado-desarrollistas inmobiliarios y limita los canales de participación. Esta situación fue expuesta como un motivo estructural del actual sistema de gobierno que genera gran parte de los conflictos urbanos. ACASM: "La Municipalidad organiza reuniones, pero estas son para 'hablar de lo que se debería hacer, pero al final no se hace nada'. Los canales de participación son muy leves y sobre todo poco democráticos, y terminan en connivencia con los desarrollistas. Principalmente por el Estado este, en forma piramidal que manda la línea y es muy difícil”.

En cuanto a las representaciones sobre las prácticas de superación de conflictos, la mayoría fue discriminada según las actuaciones de los distintos niveles de gobierno. El nivel nacional se distinguió por su escasa incidencia en la aplicación de políticas públicas de urbanización y regularización dominial y por intervenciones puntuales con intenciones de "militancia territorial”. Al gobierno provincial se lo reconoció por la política de resolución de conflictos dominiales en la regularización de asentamientos, pero se le achacó una visión asistencialista (e incluso represiva) frente a los que no accedieron a estos acuerdos y su criminalización por la "lucha de acceso a la tierra". Con respecto al gobierno municipal, se encuentra repitencia en una consideración negativa sobre sus actuaciones gubernamentales, y se percibe su rol como pobre, deficiente, reactivo y permisivo, que favorece la concentración de capital y se caracteriza por el incumplimiento normativo o la aplicación sesgada de las normas, la falta de planificación sobre la ciudad y la ausencia de programas y espacios de concertación interactoral.

DA: "Las grandes corporaciones inmobiliarias han creado un fondo fiduciario con el dinero que ha llegado desde el campo, o sea, la renta agraria que ha habido, que se ha volcado a la especulación inmobiliaria, no a resolver los problemas de vivienda que hay en la Argentina”.

EO: "Creo que no ha existido gobierno municipal en estos últimos cuatro años (risas), por lo menos en la cuestión de conflicto con la tierra no ha sido partícipe de nada”.

Las organizaciones comunitarias llegan a visualizar así al Estado, en sus diferentes niveles, como enemigo, dado que las políticas y las herramientas puestas en juego impiden el goce 


\section{La disputa por la ciudad:}

el rol de los actores sociales en la gobernanza del suelo urbano en la ciudad de Córdoba (Argentina)

efectivo de sus derechos. Al respecto, y tras las representaciones relevadas, la presión reivindicativa ("escrache") a referentes estatales y económicos se manifiesta como estrategia compartida por los actores comunitarios entrevistados para impedir o detener acciones que, según su consideración, afectarían a la comunidad de manera negativa. Los argumentos de resguardo ambiental relacionados con política de cuidado a la salud de las poblaciones se visualizan como estratégicos, dado que poseen mayores efectos sobre la lógica privada que la presión desde reivindicaciones sociales o políticas, ya que los cuestionamientos medioambientales debilitan y cuestionan socialmente sus estrategias de marketing y el "negocio privado".

ACASM: "Los escrachamos por los medios y nos sentamos a hablar para que no lo hicieran, $y$ terminaron no haciéndolo. Ellos son los que nos han permitido avanzar. Si no recurrís a los medios, si no los escrachás, no pasa nada”.

COMRENAULT: "Yo creo que... Comunidad Renault es una de las villas, digamos, que ha tratado de hacer todos los pasos de forma tranquila, de forma... todo legalmente, no hemos salido a cortar rutas, no hemos salido a hacer desastre en el centro, nada... pero bueno, yo creo que las últimas opciones son esas... para hacer escuchar”.

En cuanto a las prácticas de resolución/superación de los conflictos, se destacó como necesidad insoslayable el fomento a la participación y generación de consenso entre los tres niveles del Estado, incluyendo la participación de las organizaciones comunitarias para la concreción efectiva de políticas urbanas. Además se reiteró el pedido de un rol activo del Estado, a través de mayor planificación (no sesgada, sino orientada a la inclusión de todos los sectores sociales) y eficiencia de los organismos gubernamentales, donde prime una visión profesional y a largo plazo, que supere la mirada de cada administración particular. Junto con ello se planteó que el problema debe ser abordado "de manera integral, con perspectiva de derecho".

CEVE: "El Estado debiera avanzar en una planificación integral e integrada, de la problemática de la vivienda, del suelo, de los recursos, de las líneas de financiación, como una política pública internivel”.

DA: "Una mesa donde participen los actores afectados, o sea, los vecinos. Inclusive el capital privado de aquellos que quieren transformar la ciudad [...] el Estado tiene que tener el rol re- 
gulador, sobre todo de debate de todos los afectados, y te digo, aquellos que vienen a invertir. Tendría que haber una especie de concertación entre lo público y lo privado, sobre todo para que discutamos entre todos qué tipo de ciudad queremos”.

MOV: "[...] fundamentalmente, hay que repensar el rol del Estado con una posición más activa, que pueda regular el acceso al suelo, que pueda poner reglas más claras en cuenta al acceso, al crecimiento de los grupos desarrollistas, me parece, que de alguna forma le ponga un límite a aquel que tenga más capacidad económica se quede con la tierra y que no haya políticas de acceso para los sectores populares y sectores medios”.

NH3: "Cumpliendo la ley, planificando a futuro. Por ejemplo, hacen barrios y ni siquiera planifican cómo va a ser el tema, dónde van a tirar las aguas servidas, con pozos, si ese suelo está bien para hacer pozos, si van a pasar las cloacas, nada, nada”.

En este sentido, se visualizan diferencias entre los modos de actuación y representación de organizaciones de base social de las organizaciones ambientales o de defensa del patrimonio. Las primeras anclan su accionar en la reivindicación de derechos socio-políticos, ejercen mayor presión y reclamos a organismos provinciales y nacionales que al municipio, responden a necesidades de alcance territorial y están específicamente circunscriptas a casos y zonas de comunidades donde trabajan, y poseen escaso nivel de negociación (no se sientan a negociar, son autónomas). Las segundas estructuran su presión bajo la defensa de derechos difusos, ejercen mayor presión sobre el municipio como principal responsable; sus luchas son de mayor alcance en términos territoriales, exceden las comunidades en las que se encuentran trabajando y participan de espacios de negociación e incidencia más amplios.

Sobre las normativas conocidas en relación con las problemáticas mencionadas, se destacó un amplio repertorio de programas, políticas y normativas generales y específicas al campo de aplicación de cada organización. En este aspecto, vuelve a repetirse la sustancial diferencia con los actores estatales y económicos, lo que demuestra un trabajo interdisciplinar y de campo mucho más preciso y exhaustivo, que involucra problemáticas concretas con instrumentos y herramientas específicas coincidiendo con los sectores y conflictos que cada actor representa y ha trabajado. 


\section{La disputa por la ciudad:}

el rol de los actores sociales en la gobernanza del suelo urbano en la ciudad de Córdoba (Argentina)

En cuanto a acciones o políticas de interés, se mencionó una larga lista de acuerdo con las actividades de cada organización social. Al preguntar sobre los vínculos entre actores, las representaciones sobre quiénes son los actores más influyentes, por unanimidad se visualizan los grupos desarrollistas o las grandes corporaciones inmobiliarias de la ciudad como quienes poseen mayor influencia, seguidos por el reconocimiento del Estado provincial y municipal. Luego, en menor concurrencia, surgen otras ONG, la Universidad Nacional de Córdoba y Universidad Católica de Córdoba, sindicatos y ambientalistas.

ACASM: "Son influyentes porque la plata mueve todo, porque responden al negocio y no a la necesidad de la gente".

CEVE: "Por el manejo de recursos que tienen y la posibilidad de inversión, y el segundo por la llegada que tienen al sector político”.

MOV: "Yo creo que el actor más influyente son los grupos desarrollistas inmobiliarios. Y entendiendo más al Estado como un espacio de disputa, donde están presentes distintos intereses, hoy están siendo hegemónicos los intereses de los grupos desarrollistas. Sobre todo municipal y provincial”.

El estudio también indagó sobre las redes de actuación a fin de influir en la definición de políticas públicas. Entre los actores que las organizaciones comunitarias declaran como "con relación frecuente", en primer lugar se manifestó una cooperación fluida y nutrida entre ONG y organizaciones sociales en las que, como principales aliados, consideran a otros actores comunitarios independientemente del campo de lucha. CEVE: "Nunca solos, siempre junto a otras ONG. En el ámbito científico con organizaciones y personas que están en el tema del hábitat. Si el ámbito es más político, de políticas públicas sociales, más recostados en las instituciones del tipo ONG".

Se pueden detectar también radios de actuación por los canales de comunicación que mantienen. Los actores relacionados con movimientos políticos, de conformación nacional, mantienen comunicación con representantes en las distintas cámaras legislativas de provincia y nación. Por su parte, centros de estudiantes alcanzan contactos frecuentes con entidades nacionales, como la UNC, y el Estado nacional y provincial representados en 
diferentes secretarías. Para otras organizaciones de corte ambientalista o sociales de escala comunal, las relaciones frecuentes se limitan a los vínculos formales que establecieron en el reclamo de sus necesidades, siendo de alcance estatal — provincial y municipal—o con otras organizaciones comunitarias.

En relación con el intercambio de información, se amplía el cuadro de actores incluyendo al Estado tanto municipal como provincial en sus diferentes dependencias, las universidades (institutos de investigación y profesionales), los medios de comunicación y las redes ciudadanas.

En tanto, al hacer referencia a relaciones conflictivas, se encuentra recurrencia en mencionar al Estado como principal actor problemático. El Estado municipal aparece particularmente como fuente de conflictos, desconfianza y falta de respuestas. Los grupos desarrollistas - sector privado o empresario - también son mencionados en esta categoría, destacando la facilidad con la que operan en connivencia con el municipio. En menor coincidencia se mencionaron sindicatos y cámaras de la construcción por su actuación cómplice con las grandes corporaciones y en expresa oposición a las organizaciones comunitarias. En este sentido, es notable el rol disgregante y fragmentador que se le atribuye al Estado bajo lógicas de rédito político-económico.

MOV: "No, relaciones conflictivas se han dado en muchos barrios con grupos desarrollistas, y después hay situaciones conflictivas con el Estado, pero que en realidad expresan la puja de intereses en el interior del Estado".

SEH: "Recurrís a las Direcciones de Hábitat (provincia y municipio), en donde te encontrás con estas respuestas: que ellos te dicen 'sí, sí, sí, sí' y después no pasa nada. Sí, ahí tenemos algunas diferencias. $Y$ sobre todo, en relación al Estado, la dilación espantosa de las respuestas de las gestiones del Estado provincial y municipal. Pero terrible, así, como que no te responden nunca. Entonces eso es un problema muy grave”.

En cuanto a instituciones especializadas posibles de involucrar, los entrevistados citan una amplia gama de institutos, organizaciones y entes especializados en distintas temáticas referidas a la cuestión urbana. Sin embargo, se hace una valoración positiva de otros actores sociales, como ONG, grupos ambientalistas, institutos de investigación con una larga 


\section{La disputa por la ciudad:}

el rol de los actores sociales en la gobernanza del suelo urbano en la ciudad de Córdoba (Argentina)

trayectoria y vínculos con las organizaciones sociales, lo que afirma la descripción hecha anteriormente sobre las relaciones de confianza existentes con ellos. En menor medida, se refirieron a universidades e institutos de investigación. Solo un entrevistado (referente de CEVE) manifestó relaciones con expertos nacionales e internacionales en gestión del suelo urbano ${ }^{6}$. En su mayoría, los actores sociales visualizaron a las instituciones especializadas desde un perfil netamente académico, sin incidencia en la disputa por el derecho a la ciudad. Esta coincidencia entre los entrevistados afirma que estas instituciones tienen escasa 0 nula influencia en la definición de las políticas públicas. Al respecto se afirma que, dada la expertiz y la legitimidad con la que estas entidades cuentan, resulta necesario que sean convocadas, y se evidencia que esta ausencia es una de las grandes falencias del Estado. CEVE: "Pero nada que no salga del ámbito académico, que influya en políticas públicas, la academia no sale a disputar sentido a los gobernantes. Un buen Estado acudiría y le daría ese poder de resolución a la academia para poder influir en políticas del suelo”.

Las articulaciones con partidos políticos fueron las menos mencionadas en este aspecto, pudiendo reconocerse solo por un solo entrevistado (referente del Movimiento Evita), dada su representación política directa. Se pudo reconocer también una gran repitencia en reconocer "la muerte" de los centros vecinales como canales de participación ciudadana y de influencia política.

\section{Conclusiones}

El acceso al suelo urbano constituye uno de los factores fundamentales en el ejercicio y goce del derecho a la ciudad, por ser no solo un componente estratégico en la consecución de un hábitat digno y de inclusión en la ciudad, sino también una condición tácita para obtener sentido de ciudadanía y movilidad social (SMolKa y MulLahy, 2007).

Tanto el contexto nacional como local se caracteriza una serie de propensiones que afectan este acceso. Entre ellas se destaca una planificación urbana sesgada hacia el diseño físico y concentrada en la ciudad "legal" o "ideal" por sobre la consideración de la ciudad "real". El fuerte predominio del mercado en la definición del crecimiento urbano y su resultante en cuanto a la configuración territorial inequitativa exponen la notable autonomía de los procesos económico-financieros sobre la planificación urbana.
6. Como la Universidad General Sarmiento (Bs. As.), con referentes como el Arq. Fernández Wagner y Arq. Reese. A redes internacionales, tales como CYTED, HIC y BASIN. 
La organización y la participación de la sociedad civil asocia su lucha a la defensa de la tierra o a la satisfacción de las necesidades básicas; sin embargo, la participación ciudadana tiene escaso peso en la elaboración de políticas públicas y en la gestión urbana. Paradójicamente, la precaria presencia de las organizaciones comunitarias ha sido clave no solo en la construcción urbana de nuestras ciudades, sino en el campo de acción político, ya que logró "entorpecer" las decisiones de los gobiernos de turno, mostrando el "límite" que la sociedad pone a las provocaciones y el alto impacto que tiene la movilización popular.

El estudio realizado en torno a la gobernanza del suelo en Córdoba capital (Argentina) ha permitido evidenciar el rol que las organizaciones comunitarias tienen en la gestión del suelo, sus representaciones y redes de influencia. Mostró la "visión integral urbana” que poseen al lograr relacionar con gran solvencia y exactitud los procesos de crecimiento urbano existentes con problemáticas socio-ambientales, político-institucionales y de complicidad con intereses privados, los cuales favorecen el desarrollo territorial desigual e inequitativo vigente. Esta reflexión critica el modelo de crecimiento vigente desde una perspectiva multidimensional, así como también da significancia y valoración a la participación ciudadana como el principal aporte que estas representaciones realizan a la hora de pensar alternativas de resolución.

Sin embargo, quedan expuestas algunas falencias, como el desconocimiento o escasa valoración general otorgada a los instrumentos de regulación urbana y territorial necesarios de impulsar, al trabajo en red, a los procesos de negociación con el sector privado desde argumentos ambientales y a la manifestación mediática y la opresión reivindicativa como estrategias que resultarían potenciales de aplicar para la resolución de los conflictos urbanos a mediano y largo plazo por parte de los sectores comunitarios.

En este sentido, junto con las representaciones denunciativas manifiestas por parte de las organizaciones de la sociedad civil relevadas y sus esfuerzos incipientes de integración en torno a la lucha por una real gobernanza del suelo en la ciudad, queda expuesta una tarea pendiente: el desarrollo de una actitud autocrítica que permita una revisión y reflexión acerca de la efectividad de las experiencias de participación e incidencia y su impacto real en la calidad de las políticas urbanas y habitacionales en nuestros contextos. 


\section{La disputa por la ciudad:}

el rol de los actores sociales en la gobernanza del suelo urbano en la ciudad de Córdoba (Argentina)

\section{Bibliografía}

AZUELA, Antonio (2009). "Las reglas del desorden: habitar la metrópoli”. Reseña de Las reglas del desorden: habitar la metrópoli. Duhau E. y Giglia A. En: Revista mexicana de sociología, $\mathrm{N}^{\circ}{ }^{\circ} 71$ (4). Disponible en: http://www.scielo.org.mx/scielo.php?scrip$\mathrm{t}=\mathrm{sci}$ _arttext\&amp;pid=S018825032009000400006\&amp;lng=es\&amp;tlng=es. Fecha de consulta 1/12/2016.

BORJA, Jordi (1975) Movimientos sociales urbanos. Ediciones Siap-Planteos, Buenos Aires.

CARTA (2004) Carta Mundial por el Derecho a la Ciudad. Versión digital. Disponible en

http://www.ugr.es/ revpaz/documentacion/rpc_n5_2012_doc1.pdf. Fecha de consulta: $1 / 6 / 2016$.

CASTELLS, Manuel (2012) Redes de indignación y esperanza. Los movimientos sociales en la era de Internet. Alianza Editorial, Madrid.

DUFOUR, Dany (2009). “Gobernanza versus Gobierno”. En Cuadernos de Administración, N. ${ }^{\circ} 41$ / ene.-jun. 2009. Universidad del Valle. Colombia. Disponible en http:// www.redalyc.org/pdf/2250/225014901003.pdf.

GARGANTINI, Daniela y MARTIARENA, Miguel (comp.) (2016) Córdoba capital: tierra de conflictos. EDUCC, Córdoba, Argentina.

GUBER, Rosana (1991) El salvaje metropolitano. Editorial Legasa, Buenos Aires.

HERZER, Hilda y PÍREZ, Pedro et ál. (1993) Gestión urbana en ciudades intermedias de América Latina. UNCHS, Nairobi.

JODELET, Dense (1986) La representación social: fenómenos, concepto y teoría. En Serge Moscovici (comp.). Psicología Social II. Paidós, Barcelona.

LEFEBVRE, Henri (1969) El derecho a la ciudad. Ediciones Península, Barcelona. (1978) La producción del espacio. Ediciones Península, Barcelona. Disponible en http://dx.doi.org/10.5565/rev/papers/v3n0.880.

MoscoviCI, Serge (1979) Psicoanálisis, su imagen y su público. Huemul, Buenos Aires.

(1986) Psicología social II. Pensamiento y vida social. Psicología social y problemas sociales. Paidós, Barcelona.

MOUFFE, Chantal (2009) En torno a lo político. Fondo de Cultura Económica, Buenos Aires. 
Daniela Mariana Gargantini y Natalí Peresini

ONU-HÁBITAT. Urban governance, capacity and institutional development. [09/202/2017]. Disponible en https://www2.habitat3.org/bitcache/fd8ebaaf148654d8609926354b417f830d9a86b8?vid=586475\&disposition=inline\&op=view.

SMOLKA, Martim y MULLAHY, Laura (edit.) (2007) Perspectivas urbanas. Temas críticos en políticas de suelo en América Latina. Lincoln Institute of Land Policy, Cambridge, EE. UU.

SUBIRATS, J. y BURGUÉ, Q. (2005). “Políticas sociales metropolitanas”. En Rojas, E.; Cuadrado, J. R. y Fernández Güel, J. M. (ed.) Gobernar las metrópolis. Banco Interamericano de Desarrollo, Washington. 